\title{
Supporting Predictive Maintenance in Virtual Factory
}

\author{
Go Muan Sang, Lai Xu, and Paul de Vrieze \\ Faculty of Science and Technology, Bournemouth University, Poole, Dorset, UK \\ \{gsang, lxu, pdevrieze\}@bournemouth.ac.uk
}

\begin{abstract}
In Industry 4.0 manufacturing collaborative network, product design processes, manufacturing processes, maintenance processes should be integrated across different factories and enterprises. The collaborative manufacturing network 4.0 allows the amalgamation of manufacturing resources in multiple organizations to operate processes in a collaborative manner for reacting to the fast changes of markets or emergencies. In this paper, we propose a predictive maintenance service as a part of a virtual factory, a form of collaborative manufacturing network. Data-driven predictive maintenance service is built-in FIWARE, an industry 4.0 framework. To optimize predictive maintenance services based on different criteria within a virtual factor, such as geographical locations, similar types of machinery, or cost/time efficiency, etc., we provide our design and implementation to deal with providing better maintenance services and data exchanging across different collaborative partners with different requirements and modularizing of related functions.
\end{abstract}

Keywords: Virtual Factory, Predictive Maintenance, Maintenance Schedule, Industry 4.0, Collaborative Networks 4.0.

\section{Introduction}

Traditional monolithic manufacturing usually involves physical machines, buildings, etc., and setting the manufacturing process is generally slow and expensive [1]. Thus, it cannot deal with the challenges of dynamic market demands, competitions and short product lifecycle [2]. To overcome the challenges, modern collaborative industry is shifting towards the concept of Collaborative Networks 4.0 [2-4].

Virtual factory as one implementation of collaborative networks 4.0 and a foundational concept to future manufacturing, allows the flexible integration of manufacturing resources from different multiple organizations using emerging technologies such as cloud, sensors, IoT, etc. $[3,5,6]$. Traditional monolithic factory heavily relies on its own capabilities e.g. internal functions, physical machines, buildings, etc., whereas virtual factory allows the integration of diverse capabilities from a network of specialized domains and experts across industries collaboratively, flexibly and inexpensively regardless of their physical locations. [3, 5]. This enables the collaborative network better dealing with constant demands i.e. market, productivity, etc., since each partner firm focuses on what it does best within the network $[1,6]$. To facilitate virtual factory, a flexible platform is required $[3,5]$. 
Effective maintenance is essential to the factory collaborative network as it can impact on the collaborative network i.e. integrated processes, value and cost associated with downtime, faulty products, etc. [7, 8]. Data generated by the various processes, systems/machine equipment tools across factories operation and production offer opportunities such as data-driven analytics e.g. predictive maintenance to the collaborative network [9-12]. Flexible collaboration with other businesses is an important aspect of a virtual factory [5]. In this context, a network partner, as a service provider can offer data-driven predictive maintenance across the collaborative network i.e. manufacturers, factories, etc.

We look at how to support predictive maintenance in a collaborative network virtual factory complying Industry 4.0 standards using FIWARE and IDS, which leads to supporting flexible collaboration among different enterprises facilitating transparent data exchange and modularizing of related functions. The contributions of this work are: a) to investigate a predictive maintenance for supporting virtual factory networks, b) to present a predictive maintenance schedule using data-driven approach for virtual factory, and c) using the proposed solution to apply with a manufacturing case.

The paper is structured as follows. In Section 2, we present related work in collaborative manufacturing network 4.0 and related technologies. In Section 3 we describe the design of a reference architecture for a virtual factory with predictive maintenance service. In Section 4 and 5, we present the scheduling approaches and implementation of the predictive maintenance service in a virtual factory. The future work and conclusion are provided in Section 6.

\section{Related Work}

In this section, the relationship between collaborative networks 4.0 and virtual factory is described in Section 2.1. FIWARE industry 4.0 platform is an important industry 4.0 implementation platform. Section 2.2 presents the FIRST virtual factory reference architecture, which is adapted for our implementation. International data space is an important data storage for implementing industry 4.0 applications. IDS is introduced in Section 2.3. General work on predictive maintenance is evaluated in Section 2.4.

\subsection{Collaborative Networks 4.0 and Virtual Factory}

Collaborative networks 4.0 is driven by the amalgamation of different processes, partners, third parties, advanced analytics and machines spanning across different enterprises and organizations for collaborative value creations. Industry 4.0 drives the focus of modern manufacturing system design [2, 13]. It facilitates collaborative processes across different factories and enterprises for complex manufacturing processes. Essentially Industry 4.0 enables better control and operations to adapt in real time and in response to constant demands [14].

The concept of virtual factories derives from the expansion of virtual enterprises in the context of manufacturing [3]. Virtual factory can be seen as one of implementation of collaborative networks 4.0 in the context of Industry 4.0, and it 
allows the flexible integration of manufacturing resources in multiple organizations to manage (i.e. model, simulate, test) factory layouts and processes in a virtual environment with the support of emerging technologies such as cloud, IoT, etc. This enables the simulation of a desired factory before committing to investment and creating the actual factory in shorter time with demand-driven product lines [3].

\subsection{FIWARE Virtual Factory}

FIWARE virtual platform reference architecture in Fig. 1 maximizes cloud technology to offer smart manufacturing and digital marketplaces, especially for virtual enterprises and cross-organizations [15]. The virtual platform promotes a business ecosystem framework which supports GEs (genetic enabler components), digital asset sharing and enterprise collaboration/interoperability. These different components are the foundation of a higher-level software layer.

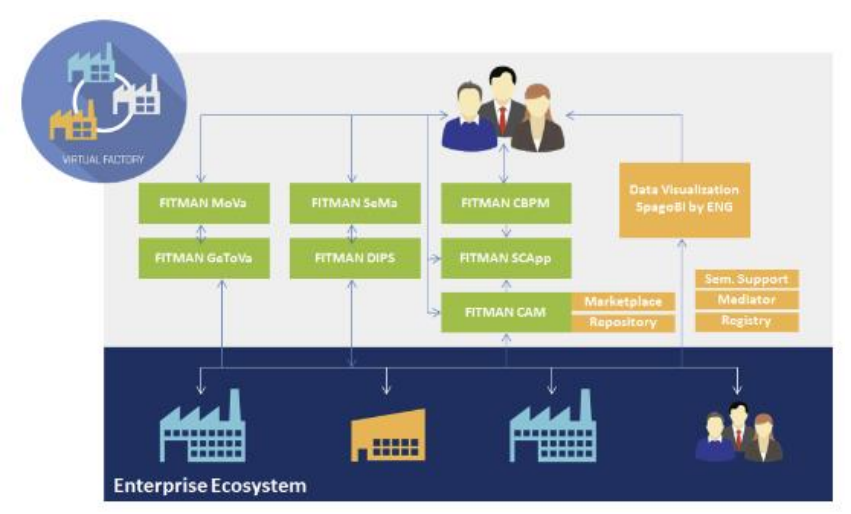

Fig. 1. FIWARE virtual factory reference architecture [15]

The collaborative assets can be managed by FITMAN CAM which supports the virtualization and management of digital assets as a platform. The supply chain and business ecosystem are supported by FITMAN SCApp. It supports digital assets such as scheduling and building processes. Collaborative business processes can be managed by FITMAN CBPM. It supports the design and execution of semanticallyannotated business processes in a web environment [15]. Data interoperability can be facilitated by FITMAN DIPS. It is a platform based on open standards such as WSMO, WSMX and supports semantic-based web service interoperability. Data mappings such as metadata and ontologies semantic matching with different OWLbased ontologies and XML schemas, can be supported by FITMAN SeMa [15]. 


\subsection{Industrial Data Space (IDS)}

The Industrial Data Space (now International data space) is a virtual data space that facilitates data exchange and association in business ecosystems using common standards and governance models [16]. Digital sovereignty of data owners is a key part of IDS, and it provides a basis for the creation and utilization of smart services and business processes [16]. It is also the important block of building a virtual factory or building a co-design and co-creation product platform [5]. The Reference Architecture Model is based on common system architecture models and standards. It utilizes a five-layer structure which states several stakeholders' concerns and viewpoints at different levels of granularity [16].

\subsection{Predictive Maintenance}

Predictive maintenance facilitates advance detection of pending failures and enables timely pre-failure interventions, using different prediction tools based on various data i.e. historical operation, condition, etc., and different machine/deep learning approaches $[8,17]$. Predictive maintenance model typically involves data collection i.e. machine tools operation/condition, data processing and modeling i.e. predictive model, and maintenance analytics i.e. maintenance schedule plan and decision making [8]. Different Industry 4.0 predictive maintenance were proposed in the research community [7, 8, 11, 18, 19]. [18] proposed a 5-level CPS architecture for smart manufacturing solutions. A flexible FIWARE predictive maintenance platform for supporting modularity and transparency in a collaborative environment is proposed by [7]. [8] designed a flexible predictive maintenance model based on FIWARE and RAMI 4.0, supporting both online/offline analytics and maintenance schedule plan. These approaches are mostly designed for the manufacturer's implementation in its own organization. Collaborative network virtual factories however involve multiple factories/organizations/partners operating in a collaborative network. Thus, a new approach is needed for the collaborative network virtual factories whereas a firm/company can provide predictive maintenance services in a modular manner.

\section{Predictive Maintenance Service Provider in a Virtual Factory}

In this paper, we present how a predictive maintenance service can be provided in a collaborative network. Predictive maintenance as a new service can collect all related data through the collaborative network as well as some data from IDS. In this section, we present a reference architecture based on FIWARE in Section 2.2, an industry 4.0 platform, which serves a base of the virtual factory or a collaborative network.

A reference architecture for supporting predictive maintenance service in a collaborative network or a virtual factory is established as presented in Fig. 2. In the virtual factory network, different enterprises, and partners (i.e. numbers of various collaborative partners such as the shopfloor, suppliers, designers, logistics, insurance, etc.) across the industry can join the network with their own services for common business objective using the FIWARE virtual factory GE components described in 
Section 2.2. And the virtual factory network can be expanded as different needs or innovative services arise.

In the reference architecture, the predictive maintenance acts as a collaborative network partner which provides predictive maintenance services to the network. Thus, it has expert knowledge and skills in big data, advanced analytics, AI, machine learning, etc., and has access to the required data such as machine operation, condition, maintenance, etc. via the network accommodated by IDS. IDS is utilized for data movement and access to gain data transparency, ownership as well as monetization. Flexibility and capabilities of big data analytics are essential aspects of operating predictive maintenance [7,11]. Thus, our previous work [7] is adopted for flexible FIWARE predictive maintenance, related big data analytics, functions and data model.

In the context of complex collaborative virtual network, predictive maintenance is essential to operating factories operation and assisting in creating effective maintenance schedule plan for decision making $[7,8,11]$. The proposed solution described in the next section addresses the consideration of data-driven approach for complex systems for optimal predictive maintenance schedule plan in virtual factories as it is still not addressed by most existing approaches [7, 8, 11, 18, 20-24].

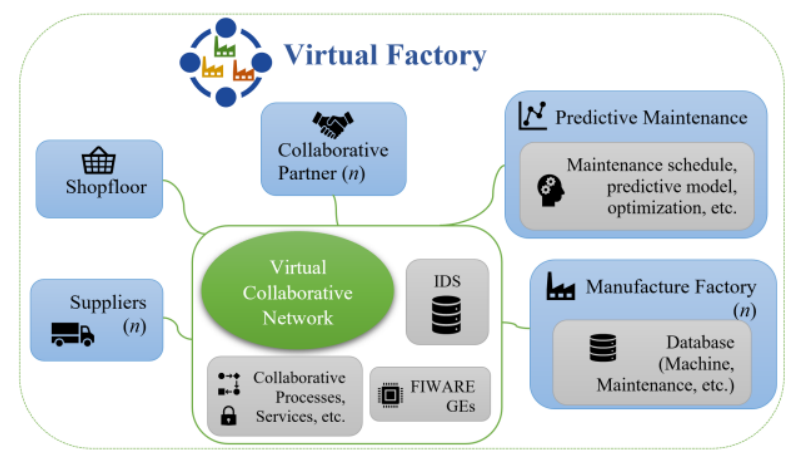

Fig. 2. A reference architecture for a virtual factory with a predictive maintenance service

\section{Scheduling for the Predictive Maintenance in Collaborative Network}

Monitoring status of different machines and different components in a machine in a collaborative network or a virtual factory is a similar activity as monitoring machines in a factory, which the related work is reviewed in Section 2.4. In this section, we focus on a schedule plan of predictive maintenance service in a collaborative network and the detailed procedure of maintenance schedule is discussed.

In general, predictive maintenance scheduling is described as an optimization process that is driven by data-driven predictions i.e. predictive model and related data i.e. 
maintenance, machine component, to assign the resources over time regarding the maintenance activities. This must satisfy a set of constraints which reflect the temporal relationships between the maintenance activities and the capacity limitations of the shared resources in a collaborative network [25]. The objective is to find optimal schedule in which a timeslot on the machines or machine components that minimizes the defined goal, is allocated for the maintenance task(s) [25].

To manage predictive maintenance scheduling in a complex collaborative context such as a maintenance service company as a part of the virtual factory, different important factors need to be considered. These factors are derived from the nature of the Industry 4.0 collaborative manufacturing setting, which includes: multiple organizations with similar machines, multiple machine components within an organization or in the collaborative network, as well as the maintenance task associated with cost and availability, which is especially for providing maintenance services within a collaborative network. The objective of data-driven optimal maintenance is to provide a maintenance schedule plan driven by predictive model incorporating with related factory maintenance data, which minimizes the overall cost related to conducting the required maintenance and thereby reducing downtime and cost. Considering the identified factors for an optimal maintenance schedule, the following procedure is established for assisting in maintenance decision process.

(a)

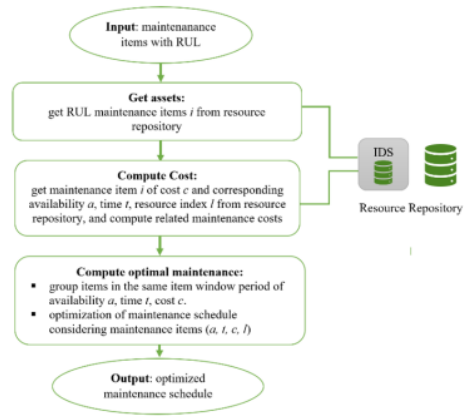

(b)

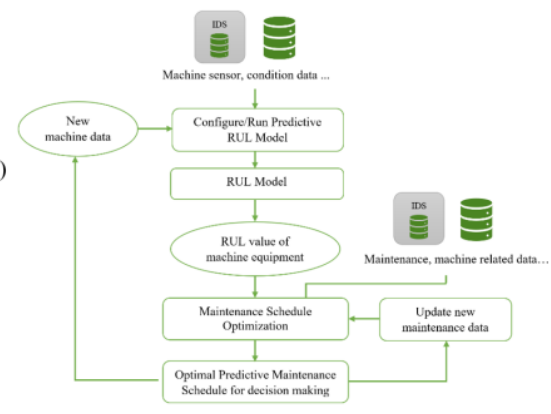

Fig. 3. (a) Algorithm 1: Optimal Maintenance Schedule (b) Procedure of Maintenance Schedule Process

Algorithm 1 in Fig. 3 (a) illustrates the optimal maintenance schedule processing considering multiple machine components driven by data-driven predictive RUL value. The input parameter, maintenance items with RUL is provided through running the predictive model. The prediction of machine equipment for remaining useful life (RUL) is considered from our previous work [7]. The input RUL value of the machine component refers to specific maintenance item with a predicted value e.g. 5 days etc.

In addition to the input maintenance items with RUL, a resource repository is used to support multiple machine component maintenance by getting any outstanding items required for maintenance within the same time window period. The resource repository accommodated by the data model from [7] is adopted. The resource repository stores the machine components and maintenance related information such as maintenance cost and availability, as well as collaborative data derived from IDS. Upon the acquisition of outstanding maintenance items, maintenance availability, 
time and cost are processed by checking any pre/plan or existing production and maintenance schedule from the resource repository against the outstanding maintenance items.

After the maintenance availability and cost are determined, maintenance items are grouped into the same time window period for optimization. Then the optimal maintenance schedule is computed using Python Pulp Optimization, and then an optimized maintenance schedule is then available for the maintenance operator/engineer assisting decision making. Section 5 provides the results with related information e.g. maintenance item, schedule, cost, in this work.

To support the dynamic nature of collaborative networks i.e. different business requirements or changes, etc., the proposed solution supports handling new data i.e. machine, maintenance, etc. as illustrated in the Maintenance Schedule Process in Fig. 3 (b). This is achieved by using new machine data, setting the RUL model, and adjusting appropriate optimization model parameters to get new RUL values. Subsequently, new maintenance schedule can be made. The next section presents the details about maintenance scenario and dataset with the corresponding results.

\section{Implementation}

In this section, a set of data are using to verify Algorithm 1 (Fig. 3 (a)). Section 5.1 explains different data related to predictive maintenance for a flexible manufacturing collaborative network and Section 5.2 presents the scheduling results based on Algorithm 1 and a predictive maintenance case in Section 5.1.

\subsection{Maintenance Case}

A maintenance case from flexible manufacturing [7] is applied for evaluating the proposed algorithm for multiple machine/component schedule plan. For example, there are three same machines in two different factories within a collaborative network. In this work, we considered 21 components from one of CNC machines of flexible manufacturing in [7]. Sensor measurement data such as temperature, vibration, energy consumption and condition (health status) of machine components, are considered as input features for the predictions [7]. The RUL values of the machine component derived from the prediction are then processed for maintenance schedule.

The maintenance dataset includes multiple factory machine components, resource index, maintenance task, timestamps, and related cost. These different data are currently collected from different sources, for analyzing the proposed maintenance schedule plan. With the proposed predictive maintenance model and maintenance schedule in Section 3 and Section 4, the different data can be easily integrated, processed, and used for different analyses after it is fully implemented.

The scenario from the case study is described in Fig. 4 (a). RUL values i.e. predicted value in day of the machine components are identified over a time window 
of 6 days period. Maintenance schedule should be planned and allocated to 6 different days period for the maintenance activities. In this scenario, 4 repairs and 1 replacement maintenance are considered. The maintenance activity i.e. repair, or replacement can also be decided by a maintenance engineer based on the predicted RUL information and other related maintenance information.

In the case of constraints, all the machine components are scheduled within their RUL period to avoid substantial maintenance and related costs such as downtime, setup, etc. The costs extracted from the case data for this model are presented in Fig. 4 (b). RUL values of the machine components are mostly utilized for the scheduling as the cost of RUL is relatively less. Group maintenance i.e. time window over 6 days with 2 available maintenance slots per day, and optimizations such as location-based based on resource index i.e. factory location/dependency are applied to reduce high value of setup/location cost. This enables the model to minimize the number of setups with associated other costs including location maintenance.
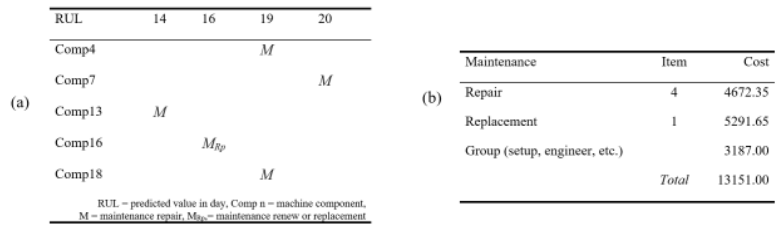

Fig. 4. (a) Machine components with RUL identified for Predictive Maintenance (b) Maintenance machine components with associated cost

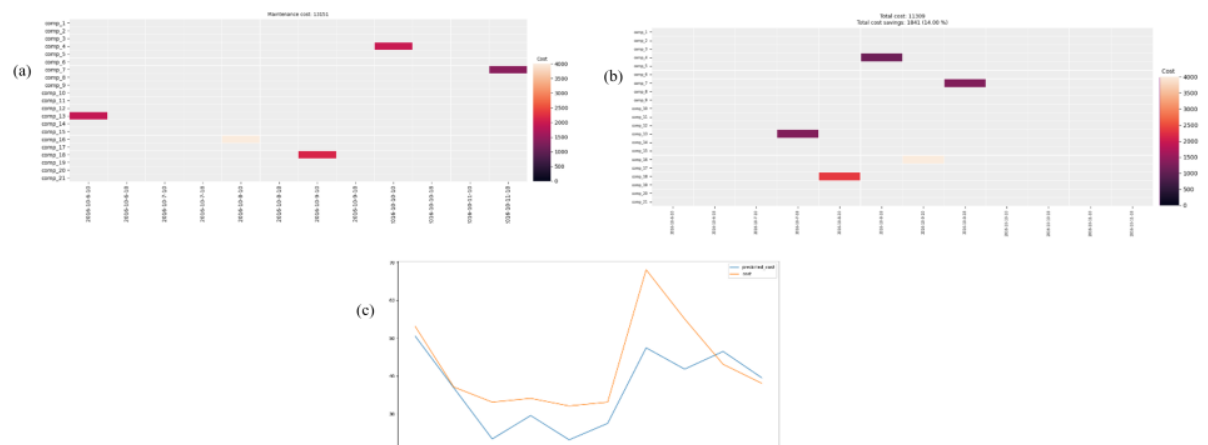

Fig. 5. Maintenance schedule with group maintenance over 6 days period (a) without optimization (b) with optimization over $14 \%$ cost saving (c) predicted cost comparison between the optimal cost and actual cost

\subsection{Scheduling Result}

The results of the proposed model are presented in Fig. 5. The subfigure (a) illustrates the normal maintenance schedule without optimization whereas the subfigure (b) represents the optimal maintenance schedule. The maintenance costs include the individual cost (repair or replacement) and setup cost which covers the engineer, downtime of each group. The optimal maintenance schedule can save over $14 \%$ of the 
expected cost based on the 6 days period window. Moreover, the maintenance prediction in subfigure (c) shows cost saving can be made over the period if maintenance activity is performed as the optimal approach suggests. Ultimately the maintenance engineer or operator can make appropriate maintenance decision based on the business needs.

\section{Conclusion}

Most predictive maintenance research focus on monitoring and scheduling maintenance tasks within one organization. Collaborative Networks 4.0 such as virtual factories are complex, dynamic and face different challenges such as a flexible platform with optimal predictive maintenance. We proposed a predictive maintenance service within a collaborative manufacturing network that offers flexible and modular components for optimizing maintenance service. A manufacturing case is used to demonstrate that predictive maintenance service can be integrated using a modular fashion into FIWARE framework and maintenance schedule plans can be created by accessing distributed data in the collaborative network. In the future, optimized models for different scenarios in different industrial sectors will be assessed.

Acknowledgments. This research is part of the FIRST project which has received funding from the European Union's Horizon 2020 research and innovation programme under the Marie Skłodowska-Curie grant agreement No. 734599.

\section{References}

1. Upton, D., McAfee, A.: The Real Virtual Factory. Harv. Bus. Rev. (1996).

2. Koren, Y., Gu, X., Guo, W.: Reconfigurable manufacturing systems: Principles, design, and future trends, (2018). https://doi.org/10.1007/s11465-018-0483-0.

3. Debevec, M., Simic, M., Herakovic, N.: Virtual factory as an advanced approach for production process optimization. Int. J. Simul. Model. (2014). https://doi.org/10.2507/IJSIMM13(1)6.260.

4. Xu, L., de Vrieze, P., Lazovik, T., Mecella, A., M., Cabri, G., Mandreoli, F., Boese, S., Bai, Y.: Overview of existing interoperability of virtual factories, D1.3, FIRST EU project H2020-MSC-RISE-2016 Ref. 6742023. Technical Report. EC. (2019).

5. Xu, L., de Vrieze, P., Yu, H.N., Keith, P., Bai, Y.: Interoperability of Virtual Factory: an Overview of Concepts and Research Challenges. Int. J. Mechatronics Manuf. Syst. (2020)

6. Sang, G.M., Xu, L., de Vrieze, P.: Mid-sized Companies in Virtual Factories a Strategy for Growth? IM\&IO. 72-75 (2020).

7. Sang, G.M., Xu, L., de Vrieze, P., Bai, Y.: Towards Predictive Maintenance for Flexible Manufacturing Using FIWARE. In: In: Dupuy-Chessa S., Proper H. (eds) Advanced Information Systems Engineering Workshops. CAiSE 2020. Lecture Notes in Business Information Processing, vol 382. Springer, Cham. pp. 17-28. Springer (2020). https://doi.org/10.1007/978-3-030-49165-9 2.

8. Sang, G.M., Xu, L., de Vrieze, P., Bai, Y.: Applying Predictive Maintenance in Flexible Manufacturing. In: In: Camarinha-Matos L.M., Afsarmanesh H., Ortiz A. (eds) Boosting Collaborative Networks 4.0. PRO-VE 2020. IFIP Advances in Information and Communication Technology, vol 598. Springer, Cham. pp. 203-212 (2020). https://doi.org/10.1007/978-3-030-62412-5_17. 
9. Thoben, K.D., Wiesner, S.A., Wuest, T.: "Industrie 4.0" and smart manufacturing-a review of research issues and application examples, (2017). https://doi.org/10.20965/ijat.2017.p0004.

10.Sang, G.M., Xu, L., de Vrieze, P.: Simplifying Big Data Analytics Systems with a Reference Architecture. In: In: Camarinha-Matos L., Afsarmanesh H., Fornasiero R. (eds) Collaboration in a Data-Rich World. PRO-VE 2017. IFIP Advances in Information and Communication Technology, vol 506. Springer, Cham. pp. 242-249 (2017). https://doi.org/10.1007/978-3-319-65151-4_23.

11.Sang, G.M., Xu, L., de Vrieze, P., Bai, Y., Pan, F.: Predictive Maintenance in Industry 4.0. In: Proceedings of the 10th International Conference on Information Systems and Technologies. pp. 1-11. ACM, New York, NY, USA (2020). https://doi.org/10.1145/3447568.3448537.

12.Sang, G.M., Xu, L., de Vrieze, P.: A reference architecture for big data systems. In: 2016 10th International Conference on Software, Knowledge, Information Management \& Applications (SKIMA). pp. $370-375$. https://doi.org/10.1109/SKIMA.2016.7916249.

13.Zezulka, F., Marcon, P., Vesely, I., Sajdl, O.: Industry 4.0 - An Introduction in the phenomenon. IFAC-PapersOnLine. (2016). https://doi.org/10.1016/j.ifacol.2016.12.002.

14.Porter, M.E., Heppelmann, J.E.: How smart, connected products are transforming competition, (2014).

15.FIWARE: FIWARE Virtual Factory Reference Architecture, https://www.fiware4industry.com/virtual-factory-reference-architecture/, last accessed $2021 / 04 / 15$

16.Otto, B., Steinbuß, S., Teuscher, A., Lohmann, S.: Reference architecture model Version 3.0, International Data Space Association. (2019).

17.Mobley, R.K.: An Introduction to Predictive Maintenance (Second Edition). (2002). https://doi.org/10.1016/B978-075067531-4/50018-X.

18.Lee, J., Bagheri, B., Kao, H.A.: A Cyber-Physical Systems architecture for Industry 4.0based manufacturing systems. Manuf. Lett. (2015). https://doi.org/10.1016/j.mfglet.2014.12.001.

19.Wang, L.: Machine availability monitoring and machining process planning towards Cloud manufacturing. CIRP J. Manuf. Sci. Technol. (2013). https://doi.org/10.1016/j.cirpj.2013.07.001.

20.Wang, H.: A survey of maintenance policies of deteriorating systems. Eur. J. Oper. Res. (2002). https://doi.org/10.1016/S0377-2217(01)00197-7.

21.Chan, G.K., Asgarpoor, S.: Optimum maintenance policy with Markov processes. Electr. Power Syst. Res. (2006). https://doi.org/10.1016/j.epsr.2005.09.010.

22.Nicolai, R.P., Dekker, R.: A review of multi-component maintenance models. In: Proceedings of the European Safety and Reliability Conference 2007, ESREL 2007 - Risk, Reliability and Societal Safety (2007).

23.Dekker, R., Wildeman, R.E., Van Der Duyn Schouten, F.A.: A review of multi-component maintenance models with economic dependence. Math. Methods Oper. Res. (1997). https://doi.org/10.1007/BF01194788.

24.Van Horenbeek, A., Pintelon, L.: A dynamic predictive maintenance policy for complex multi-component systems. Reliab. Eng. Syst. Saf. (2013). https://doi.org/10.1016/j.ress.2013.02.029.

25.Pinedo, M.L.: Scheduling. Springer International Publishing, Cham (2016). https://doi.org/10.1007/978-3-319-26580-3. 